\title{
EVALUACIÓN DE EFECTOS DE INTERACCIÓN EN RESISTENCIAS INELÁSTICAS
}

\author{
Luis E. Pérez-Rocha ${ }^{(1)}$ y Javier Avilés ${ }^{(2)}$
}

\begin{abstract}
RESUMEN
Se evalúan los efectos de interacción suelo-estructura en las resistencias requeridas para sistemas inelásticos, incluyendo la interacción cinemática e inercial. Se estudia un sistema sencillo de interacción representativo de edificios diseñados conforme a reglamento. El enfoque del oscilador de reemplazo, usado en la práctica para tomar en cuenta los efectos elásticos de interacción, se extiende para considerar el comportamiento no lineal de la estructura. Éste está definido por el periodo, amortiguamiento y ductilidad efectivos del sistema. Se presentan soluciones numéricas para estructuras desplantadas en un estrato de suelo sobre un semiespacio elástico, ante la propagación vertical de ondas de cortante. Se calculan espectros elásticos e inelásticos para la condición de base flexible y se comparan con los correspondientes a la condición de base rígida, con el propósito de determinar cómo difieren los efectos de interacción entre sistemas lineales y no lineales. También se examinan los factores de reducción de resistencia que pueden emplearse para estimar las resistencias inelásticas requeridas a partir de las resistencias elásticas correspondientes. Los resultados presentados suministran una valiosa información sobre los efectos inelásticos de interacción, que puede aprovecharse para formular reglas de diseño para edificios apoyados flexiblemente.
\end{abstract}

\section{SUMMARY}

The effects of soil-structure interaction in inelastic systems are evaluated, including both kinematic and inertial interaction. A single interacting system representative of code-designed buildings is investigated. The replacement oscillator approach used in practice to account for the elastic interaction effects is extended to consider the nonlinear behavior of the structure. An inelastic replacement oscillator defined by an effective ductility along with the effective period and damping of the system for the elastic condition is proposed. Numerical solutions are given for structures with embedded foundation in a soil layer over elastic bedrock, subjected to vertically propagating shear waves. Computed elastic and inelastic response spectra for the flexible-base structure are compared to those for the fixed-base condition, in order to determine how interaction effects differ between linear and nonlinear systems. Strength reduction factors that may be used to estimate inelastic required strengths starting from their elastic counterparts are also examined. The ensuing results supply valuable information from which the inelastic interaction effects in flexibly supported structures can be readily assessed for engineering applications.

Artículo recibido el 17 de septiembre de 2002 y aprobado para su publicación el 9 de septiembre de 2003. Se aceptarán comentarios y/o discusiones hasta cinco meses después de su publicación.

(1) Centro de Investigación Sísmica, Carretera al Ajusco 203, Tlalpan 14200, México, DF., perezrocha63@hotmail.com

(2) Instituto Mexicano de Tecnología del Agua, Jiutepec 62550, Morelos, México, javiles@tajin.imta.mx 


\section{INTRODUCCIÓN}

Aunque los efectos de interacción suelo-estructura han sido el propósito de numerosas investigaciones en el pasado, generalmente en ellas se ha excluido el comportamiento no lineal de la estructura. Jennings y Bielak (1973) y Veletsos y Meek (1974) hicieron los primeros estudios de interacción con sistemas elásticos, usando una analogía con un oscilador simple equivalente. Ellos mostraron que los efectos de interacción inercial pueden ser suficientemente aproximados modificando simplemente el periodo fundamental y el amortiguamiento asociado de la estructura con base rígida. Después de estas investigaciones, el incremento en el periodo natural y el cambio en el amortiguamiento debidos a la flexibilidad del suelo y a la radiación de ondas, respectivamente, han sido extensamente estudiados por varios autores (Bielak, 1975; Wolf, 1985; Avilés y Pérez-Rocha, 1996), empleando como excitación en la base un movimiento armónico de amplitud constante. Con la misma analogía del oscilador equivalente, los efectos de interacción cinemática en las propiedades dinámicas relevantes de la estructura se han evaluado para diferentes tipos de ondas sísmicas incidentes (Todorovska y Trifunac, 1992; Avilés y PérezRocha, 1998; Avilés et al., 2002).

En su forma actual, el enfoque del oscilador de reemplazo es estrictamente aplicable sólo para tomar en cuenta los efectos elásticos de interacción. No obstante que no se considera el comportamiento inelástico de la estructura, este enfoque ha sido adoptado en normas de diseño sísmico avanzadas (ATC, 1984; FEMA, 1994) por la conveniencia de usar espectros de respuesta de campo libre en combinación con el periodo y amortiguamiento efectivos del sistema. Puesto que los efectos de interacción pueden diferir apreciablemente entre sistemas elásticos e inelásticos, las recomendaciones sobre interacción que aparecen en la mayoría de los actuales reglamentos, basadas en estudios de respuesta elástica, podrían no resultar apropiadas para el diseño sísmico de edificios típicos. Como es sabido, para estas estructuras se espera la ocurrencia de deformaciones considerablemente mayores que el límite de fluencia durante temblores intensos.

Los primeros estudios de la respuesta inelástica de estructuras con apoyo indeformable corresponden a Veletsos et al. (1965) y Veletsos (1969), quienes examinaron osciladores de un grado de libertad, y a Veletsos y Vann (1971) que analizaron sistemas de varios grados de libertad. Ellos obtuvieron reglas aproximadas simples que relacionan la deformación máxima y la resistencia de fluencia de estructuras no lineales con los valores correspondientes de la estructura lineal asociada. Para ello, emplearon ondículas sencillas y temblores de banda ancha como excitación. No existen relaciones similares que tomen en cuenta la flexibilidad del suelo, mediante las cuales pueda estimarse la respuesta máxima de estructuras inelásticas a partir de un análisis lineal de interacción. Se requiere de una investigación más completa para mejorar el entendimiento de los efectos de interacción en sistemas no lineales. Los resultados pueden servir de base para la formulación de criterios de diseño sísmico para edificios apoyados flexiblemente.

Veletsos y Verbic (1974) examinaron brevemente la respuesta transitoria de una estructura elastóplastica apoyada en la superficie de un semiespacio. Ellos sugirieron que el comportamiento no lineal reduce la rigidez de la estructura respecto al suelo y, por tanto, decrecen los efectos de interacción suelo-estructura. Basado en la respuesta armónica de una 
estructura con comportamiento histerético bilineal apoyada en la superficie de un semiespacio viscoelástico, Bielak (1978) ha mostrado que la deformación estructural resonante puede ser significativamente más grande que la que resultaría si el medio de soporte fuera rígido. Un estudio reciente de Rodríguez y Montes (1998) ha señalado que los efectos de interacción en la Ciudad de México son en general más importantes para sistemas elásticos que para inelásticos, conclusión similar a la que previamente habían llegado Bazán et al. (1992) para otros escenarios de interacción. Estos autores también han sugerido que la respuesta inelástica de edificios sobre suelo blando puede aproximarse usando espectros de respuesta de base rígida junto con el periodo efectivo del sistema suelo-estructura, despreciando con ello los efectos de interacción en el amortiguamiento y la ductilidad estructurales. Para edificios diseñados conforme a reglamento, sin embargo, hace falta desarrollar reglas prácticas que permitan estimar fácilmente la resistencia requerida y el desplazamiento esperado de estructuras inelásticas con base flexible a partir de los valores correspondientes de estructuras elásticas con base rígida. El propósito de este estudio está dirigido a satisfacer en parte esta necesidad.

Los efectos de interacción suelo-estructura en la ductilidad no han sido suficientemente esclarecidos hasta el momento. Los principales objetivos que aquí se persiguen son: (1) formular un enfoque eficiente para tomar en cuenta los efectos inelásticos de interacción en sistemas simples excitados sísmicamente; (2) evaluar la influencia de los principales parámetros involucrados y la importancia relativa de los efectos elásticos e inelásticos de interacción; y (3) desarrollar información que pueda ser usada para estimar la respuesta inelástica de edificios típicos excitados por el movimiento efectivo de la cimentación, en términos de la respuesta inelástica de un oscilador de reemplazo excitado por el movimiento de campo libre en la superficie.

El sistema suelo-estructura considerado en este estudio está formado por una estructura elastoplástica de un nivel apoyada en un cimiento rígido enterrado en un estrato de espesor constante sobre un semiespacio homogéneo. Este sistema acoplado, aunque simple, proporciona resultados de gran utilidad para aplicaciones de ingeniería, ya que satisface varios requerimientos sugeridos por los reglamentos de construcción. Además, se incorporan los efectos de interacción cinemática debidos a la diferencia entre la excitación efectiva de entrada y el movimiento de campo libre, para el caso de cimentaciones enterradas ante la incidencia vertical de ondas de corte. Por las características de estas ondas, la excitación efectiva de entrada consiste en un componente horizontal y otro de cabeceo, excepto para el caso de cimentaciones superficiales donde dicha excitación es igual al componente horizontal del movimiento de campo libre.

Los conceptos presentados en este trabajo se desarrollaron con referencia al enfoque del oscilador de reemplazo comúnmente empleado en las normas de diseño sísmico para considerar los efectos elásticos de interacción. Aquí se propone un oscilador de reemplazo no lineal, caracterizado por una ductilidad efectiva junto con el periodo y amortiguamiento efectivos del sistema para la condición elástica. Se muestra que modificando el factor de ductilidad, el periodo natural y el amortiguamiento de la estructura con base rígida, se obtiene una herramienta práctica y fidedigna para tomar en cuenta completamente los efectos inelásticos de interacción. Se calcularon resultados numéricos para una gran variedad de configuraciones de sistemas sueloestructura, tomando como movimiento de control el gran terremoto de Michoacán de 1985 
registrado en dos sitios representativos de las zonas de transición y del lago de la Ciudad de México. Si bien los efectos de interacción dependen del contenido de frecuencias del movimiento incidente, el uso de este movimiento de control es aceptable por tratarse de un evento representativo de los grandes temblores de subducción, los cuales típicamente excitan la frecuencia dominante del terreno en sitios de suelo blando. Finalmente, se compararon los resultados para las condiciones de base rígida y flexible, considerando los efectos del comportamiento lineal y no lineal de la estructura, con el propósito de identificar cómo se afecta la respuesta estructural por interacción y qué tan importantes son los efectos de interacción en sistemas inelásticos.

\section{FORMULACIÓN DEL PROBLEMA}

\section{Sistema suelo-estructura}

El sistema suelo-estructura estudiado se ilustra en la fig. 1. Consiste en una estructura de un nivel apoyada en una cimentación rígida que está embebida en un estrato viscoelástico de espesor constante que sobreyace a un semiespacio viscoelástico uniforme. La estructura se caracteriza por la altura $H_{e}$, masa $M_{e}$ y momento de inercia de masa $J_{e}$ alrededor de un eje centroidal horizontal. El periodo natural y la fracción de amortiguamiento de la estructura para las condiciones elástica y de base rígida están dados por

$$
\begin{aligned}
& T_{e}=2 \pi \sqrt{\frac{M_{e}}{K_{e}}} \\
& \zeta_{e}=\frac{C_{e}}{2 \sqrt{K_{e} M_{e}}}
\end{aligned}
$$

donde $C_{e}$ y $K_{e}$ son, respectivamente, el amortiguamiento viscoso y la rigidez inicial de la estructura con base rígida. La cimentación es circular, perfectamente adherida al suelo, tiene radio $R$, profundidad de enterramiento $D$, masa $M_{c}$ y momento de inercia de masa $J_{c}$ alrededor de un eje centroidal horizontal. El estrato se caracteriza por el espesor $H_{s}$, relación de Poisson $v_{s}$, densidad de masa $\rho_{s}$, velocidad de ondas de corte $\beta_{s}$ y factor de amortiguamiento histerético $\zeta_{s}$. Las correspondientes propiedades materiales del semiespacio se definen por $v_{o}$, $\rho_{o}, \beta_{o} \mathrm{y} \zeta_{o}$.

Para estimar la máxima respuesta de edificios de varios pisos es aceptable suponer que la interacción suelo-estructura afecta sólo el componente de respuesta debido al modo fundamental de vibración (Jennings y Bielak, 1973). En este caso, es necesario interpretar los parámetros del oscilador simple como aquéllos del edificio de varios pisos vibrando en su modo fundamental de base rígida. 


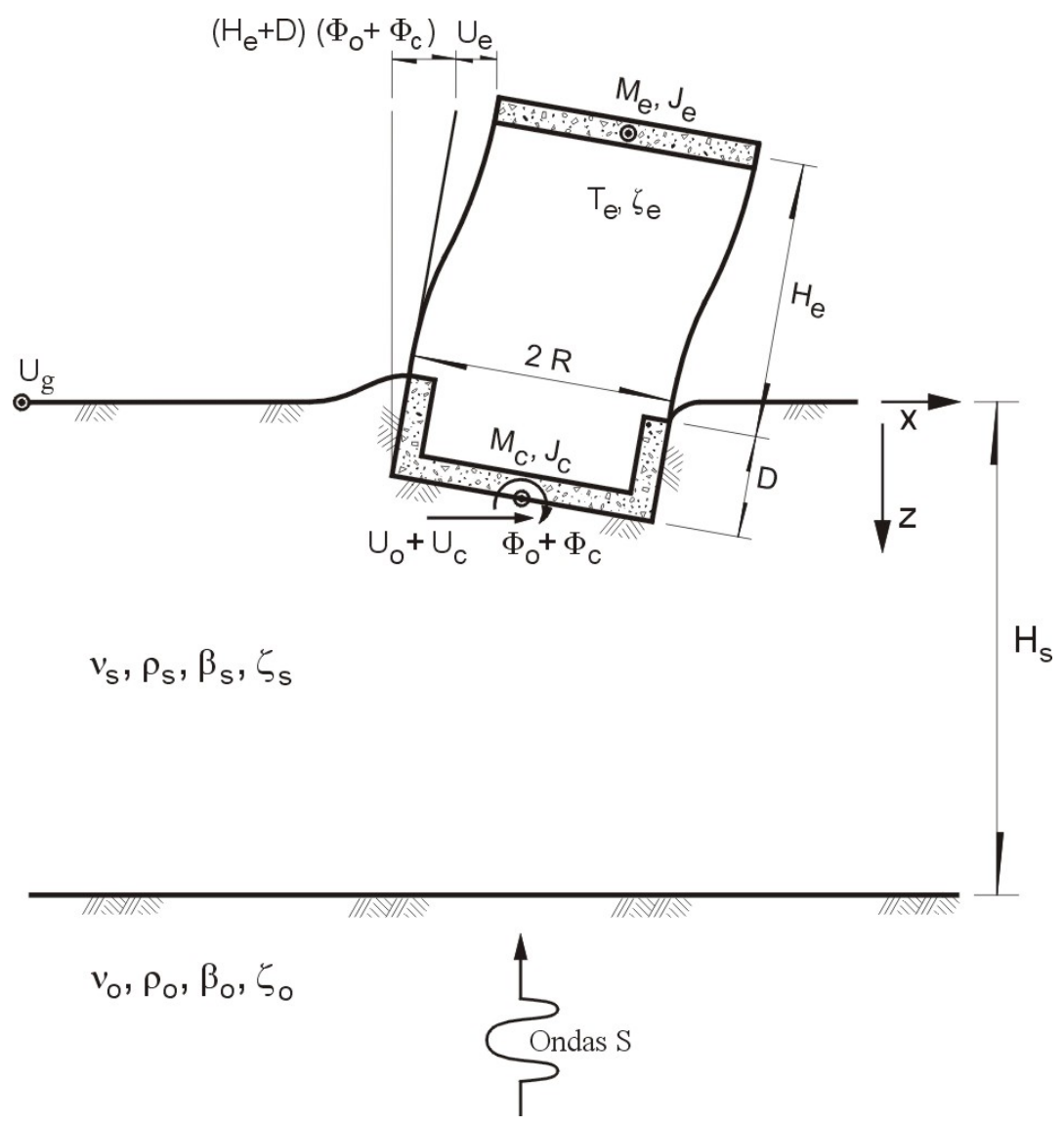

Figura 1. Estructura elastoplástica apoyada en una cimentación rígida enterrada en un estrato que descansa sobre un semiespacio bajo incidencia vertical de ondas de cortante.

Es bien sabido que el alargamiento del periodo y el aumento del amortiguamiento por interacción con el suelo son similares a los efectos del comportamiento no lineal de la estructura. Estos últimos dependen del tipo de comportamiento histerético. En este trabajo, la relación fuerza-deformación para el elemento resistente se considera de tipo elastoplástico, ya que ello permite derivar un factor de ductilidad equivalente para incluir los efectos de interacción. En la fig. 2 se ilustran los diagramas de resistencia de la estructura elastoplástica y de una estructura elástica asociada que tiene el mismo periodo natural y fracción de amortiguamiento iniciales de la estructura real. Para la estructura elastoplástica, la resistencia de fluencia se denota por $R_{y}$, la deformación de fluencia por $U_{y}$ y la deformación máxima absoluta por $U_{m}$. El factor de ductilidad se define entonces como

$$
\mu_{e}=\frac{U_{m}}{U_{y}}
$$

Para estructuras rígidamente apoyadas, en diseño sísmico usualmente se aplica la regla de igual desplazamiento. De acuerdo con esta regla clásica, la deformación máxima absoluta de la 
estructura inelástica es similar a la que se tiene en la estructura elástica, excepto para periodos cortos. Esto permite escribir el factor de ductilidad de la siguiente forma:

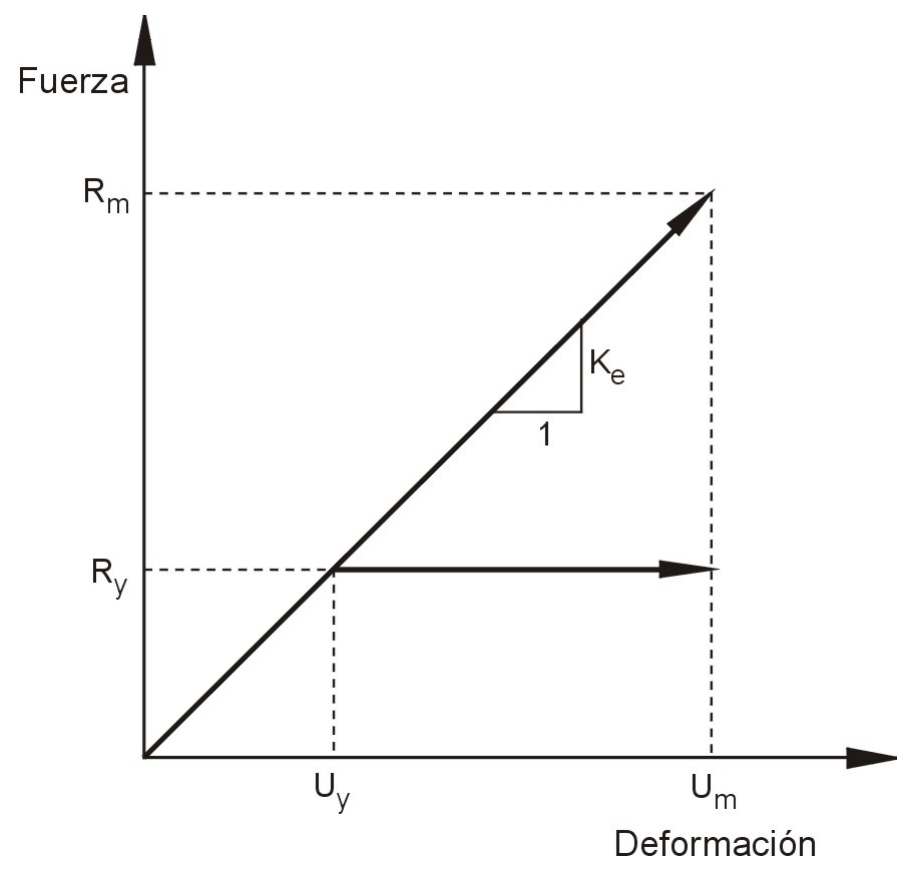

Figura 2. Relaciones fuerza-deformación para la estructura elastoplástica real y la estructura elástica asociada con el mismo periodo y amortiguamiento iniciales.

$$
\mu_{e}=\frac{R_{m}}{R_{y}}
$$

donde $R_{m}$ es la resistencia elástica. Si esta resistencia se conoce, la resistencia inelástica requerida para una ductilidad dada se calcula a partir de la ec. 4, la cual es válida para el caso de suelo firme. También se han desarrollado reglas empíricas por Miranda (1993) y Ordaz y PérezRocha (1998) para sitios de suelo blando, que dependen de la relación entre los periodos de la estructura y el sitio.

\section{Ecuaciones de equilibrio}

El sistema suelo-estructura está sujeto a la incidencia vertical de ondas de corte, propagándose en la dirección del eje $z$, con movimiento de las partículas en la dirección del eje $x$. El desplazamiento horizontal en la superficie del terreno generado por el movimiento de campo libre se denota por $U_{g}$. La presencia de la cimentación modifica el movimiento de campo libre por la generación de ondas difractadas, resultando en un movimiento efectivo de entrada con un componente horizontal y otro de cabeceo, denotados por $U_{o}$ y $\Phi_{o}$, respectivamente. En este caso, no se genera el componente de torsión. 
Los grados de libertad del sistema cimentación-estructura son el desplazamiento relativo de la estructura $U_{e}$, el desplazamiento de la cimentación $U_{c}$ relativo al movimiento horizontal de entrada $U_{o}$, y el cabeceo de la cimentación $\Phi_{c}$ relativo al movimiento rotacional de entrada $\Phi_{o}$. Las ecuaciones de equilibrio del sistema acoplado en el dominio del tiempo se pueden escribir en forma matricial como

$$
\mathbf{M}_{s} \ddot{\boldsymbol{\delta}}_{s}(t)+\mathbf{P}_{s}(t)=-\mathbf{M}_{o} \ddot{U}_{o}(t)-\mathbf{J}_{o} \ddot{\Phi}_{o}(t)
$$

donde $\boldsymbol{\delta}=\left\{U_{e}, U_{c}, \Phi_{c}\right\}^{T}$ es el vector de desplazamientos del sistema; el punto superpuesto denota diferenciación con respecto al tiempo $t$. Por su parte, $\mathbf{M}_{o} \mathrm{y} \mathbf{J}_{o}$ son los vectores de carga

$$
\begin{gathered}
\mathbf{M}_{o}=\left\{\begin{array}{c}
M_{e} \\
M_{e}+M_{c} \\
M_{e}\left(H_{e}+D\right)+M_{c} E
\end{array}\right\} \\
\mathbf{J}_{o}=\left\{\begin{array}{c}
M_{e}\left(H_{e}+D\right) \\
M_{e}\left(H_{e}+D\right)+M_{c} E \\
M_{e}\left(H_{e}+D\right)^{2}+M_{c} E^{2}+J_{e}+J_{c}
\end{array}\right\}
\end{gathered}
$$

$\mathbf{M}_{s}$ es la matriz de masas del sistema

$$
\mathbf{M}_{s}=\left[\begin{array}{ccc}
M_{e} & M_{e} & M_{e}\left(H_{e}+D\right) \\
M_{e} & M_{e}+M_{c} & M_{e}\left(H_{e}+D\right)+M_{c} E \\
M_{e}\left(H_{e}+D\right) & M_{e}\left(H_{e}+D\right)+M_{c} E & M_{e}\left(H_{e}+D\right)^{2}+M_{c} E^{2}+J_{e}+J_{c}
\end{array}\right]
$$

y $\mathbf{P}_{s}$ es el vector de fuerzas internas del sistema

$$
\mathbf{P}_{s}(t)=\left\{\begin{array}{l}
P_{e}(t) \\
\mathbf{P}_{c}(t)
\end{array}\right\}
$$

En la deducción de las ecuaciones de equilibrio del sistema, el centro de masa de la cimentación se ha localizado a la mitad de la profundidad de enterramiento, $E=D / 2$, lo cual es poco importante para fines prácticos.

Por otro lado, en la ec. 9 se tiene que

$$
P_{e}(t)=C_{e} \dot{U}_{e}(t)+R_{e}(t)
$$


donde $R_{e}$ es la fuerza restauradora de la estructura, y $\mathbf{P}_{c}$ es el vector de fuerzas de interacción del suelo actuando sobre la cimentación, dadas por la integral de convolución

$\mathbf{P}_{c}(t)=\int_{0}^{t} \mathbf{K}_{c}(t-\tau)\left\{\begin{array}{l}U_{c}(\tau) \\ \Phi_{c}(\tau)\end{array}\right\} d \tau$

donde $\mathbf{K}_{c}$ representa la matriz de rigidez dinámica del suelo en el dominio del tiempo, es decir, las fuerzas requeridas para producir desplazamientos impulsivos unitarios de la cimentación. Haciendo uso de la transformada inversa de Fourier, esta matriz se puede calcular como

$\mathbf{K}_{c}(t)=\frac{1}{2 \pi} \int_{-\infty}^{+\infty} \mathbf{K}_{c}^{*}(\omega) e^{i \omega t} d \omega$

en la que $\omega$ es la frecuencia circular de excitación, $i=\sqrt{-1}$ la unidad imaginaria y

$\mathbf{K}_{c}^{*}=\left[\begin{array}{cc}\widetilde{K}_{h h}(\omega) & \widetilde{K}_{h r}(\omega) \\ \widetilde{K}_{h r}(\omega) & \widetilde{K}_{r r}(\omega)\end{array}\right]$

representa la matriz de rigidez dinámica del suelo en el dominio de la frecuencia, es decir, las fuerzas requeridas para producir movimientos armónicos unitarios de la cimentación. En la ec. 13 se tiene que $\widetilde{K}_{h h}, \widetilde{K}_{r r}$ y $\widetilde{K}_{h r}$ son las funciones de impedancia para los modos de vibración horizontal, rotacional y acoplado, respectivamente. Estas son funciones complejas que se expresan como

$\widetilde{K}_{m n}(\omega)=K_{m n}(\omega)+i \omega C_{m n}(\omega) ; \quad m, n=h, r$

donde $K_{m n}$ y $C_{m n}$ representan los resortes lineales y amortiguadores viscosos, respectivamente, mediante los que se reemplaza el suelo para cada modo de vibración de la cimentación. Los resortes reflejan la rigidez e inercia del suelo, mientras que los amortiguadores reflejan los amortiguamientos material y geométrico debidos al comportamiento histerético y a la radiación de ondas, respectivamente.

\section{Método de solución}

El análisis de interacción suelo-estructura puede efectuarse en el dominio de la frecuencia usando funciones de impedancia armónicas, o bien en el dominio del tiempo usando funciones de impedancia impulsivas. El análisis en el dominio de la frecuencia, sin embargo, no es práctico para estructuras que se deforman inelásticamente. Por otro lado, el análisis en el dominio del tiempo puede realizarse mediante el uso de modelos para la cimentación que sean independientes de la frecuencia, es decir, con resortes y amortiguadores constantes calculados como lo indican 
Wolf y Somaini (1986). Con esta simplificación, se evita la integral de convolución que describe las fuerzas de interacción del suelo, y así el procedimiento de integración de las ecuaciones de equilibrio se aplica como en el caso de base rígida. Los cálculos usualmente se ejecutan con los valores de rigidez para frecuencia cero y de amortiguamiento para frecuencia infinita. Esto se conoce como la aproximación doblemente asintótica (Wolf, 1988) y es, en efecto, asintóticamente exacta para bajas y altas frecuencias. Para mejorar la aproximación, los resortes y amortiguadores pueden evaluarse para otra frecuencia específica, por ejemplo la frecuencia del sistema para la condición elástica, como se ha hecho en esta investigación, o bien pueden promediarse dentro del intervalo de frecuencias de interés.

Para calcular la respuesta no lineal de la estructura apoyada flexiblemente, se hizo uso de un esquema de integración paso a paso en el dominio de tiempo basado en el método de Newmark. Los detalles de este método aplicado a estructuras de base rígida es bien conocido. En este estudio se calculan espectros de respuesta de ductilidad constante. Con ellos se suministra la resistencia de fluencia de estructuras con apoyo flexible, como función del periodo natural de base rígida, que se requiere para limitar la demanda de ductilidad a un factor de ductilidad prescrito. Como la forma de los espectros de respuesta depende fuertemente de los efectos de sitio, la excitación sísmica tomada como movimiento de control debe ser consistente con las condiciones locales del subsuelo.

Los espectros de respuesta de ductilidad constante se calcularon por iteración sobre la resistencia estructural $R_{y}$ hasta que la demanda de ductilidad dada por la ec. 3 fuera igual a la ductilidad disponible especificada. En esta ecuación, $U_{y}=R_{y} / K_{e}$ y $U_{m}$ es la deformación máxima absoluta $U_{e}^{\max }$ calculada con la ec. 5. El proceso de iteración se detiene cuando la diferencia entre las ductilidades calculada y objetivo se considera satisfactoria para propósitos de ingeniería. La tolerancia fijada en este estudio fue 1\%. Se tomaron las debidas precauciones cuando la demanda de ductilidad no se incrementa monotónicamente conforme decrece la resistencia de fluencia de la estructura. En este caso existe más de una resistencia que produce una demanda de ductilidad igual a la ductilidad objetivo, pero sólo la resistencia más grande es de interés para diseño sísmico.

\section{Parámetros del sistema}

Para el sistema suelo-estructura investigado, los parámetros adimensionales que convenientemente se han definido para llevar a cabo la evaluación de los efectos de interacción son los siguientes: la relación de masas entre la cimentación y la estructura, $M_{c} / M_{e}$; la relación de momentos de inercia de masa entre la cimentación y la estructura, $J_{c} / J_{e}$; la relación de densidades de masa entre el estrato y la roca basal, $\rho_{s} / \rho_{o}$; la relación entre las velocidades de propagación de ondas de corte del suelo y la roca basal $\beta_{s} / \beta_{o}$; la relación de esbeltez de la estructura, $H_{e} / R$; la relación de enterramiento de la cimentación, $D / R$; el espesor relativo del 
estrato, $H_{s} / R$; y el contraste de rigidez entre la estructura y el suelo, $H_{e} / \beta_{s} T_{e}$. Este último es el parámetro que controla la interacción suelo-estructura.

Nótese que el parámetro de onda $R / \beta_{s}$, conocido como tiempo de tránsito $\mathrm{y}$ frecuentemente empleado para caracterizar la flexibilidad del suelo, está relacionado con el periodo fundamental y el espesor relativo del estrato por

$$
\frac{R}{\beta_{s}}=\frac{T_{s}}{4} \frac{R}{H_{s}}
$$

Para depósitos de suelo estratificado, los parámetros $T_{s}$ y $\beta_{s}$ deben interpretarse como el periodo dominante y la velocidad promedio del sitio, respectivamente.

\section{OSCILADOR DE REEMPLAZO INELÁSTICO}

\section{Periodo y amortiguamiento efectivos del sistema}

Los efectos elásticos de interacción se expresan normalmente mediante cambios en el periodo natural $T_{e} \mathrm{y}$ en la fracción de amortiguamiento $\zeta_{e}$ de la estructura con base rígida. Los parámetros resultantes $\widetilde{T}_{e}$ y $\widetilde{\zeta}_{e}$ se definen como el periodo efectivo y la fracción de amortiguamiento efectivo del sistema suelo-estructura, respectivamente. Estos pueden determinarse empleando una analogía entre el sistema acoplado excitado por el movimiento de entrada en la base de la cimentación y un oscilador de reemplazo excitado por el movimiento de campo libre en la superficie del terreno. La masa del oscilador se toma igual a la de la estructura, despreciando la masa de la cimentación. Con base en esta analogía, el periodo y amortiguamiento efectivos del sistema se obtienen tal que, bajo excitación armónica, el periodo resonante y la fuerza restauradora máxima del sistema real sean iguales a los del oscilador equivalente. La formulación y validación de este procedimiento para calcular el periodo y amortiguamiento estructurales modificados tanto por la interacción cinemática como por la inercial ya se ha publicado (Avilés y Pérez-Rocha, 1998). Los parámetros $\widetilde{T}_{e}$ y $\widetilde{\zeta}_{e}$ se miden directamente de la función de transferencia del sistema para la condición elástica, la cual se define como $Q(\omega)=\omega_{e}^{2} U_{e}(\omega) / \omega^{2} U_{g}(\omega)$, siendo $\omega_{e}$ la frecuencia fundamental de base rígida. Una vez que se determina la amplitud de la función de transferencia, el periodo y amortiguamiento efectivos del sistema se calculan aplicando las siguientes expresiones:

$$
\begin{aligned}
& \widetilde{T}_{e}=\sqrt{1-2 \widetilde{\zeta}_{e}^{2}} T_{r e s} \\
& \widetilde{\zeta}_{e}=\frac{1}{\sqrt{2}}\left(1-\sqrt{\frac{Q_{r e s}^{2}-1}{Q_{\text {res }}^{2}}}\right)^{1 / 2}
\end{aligned}
$$


donde $T_{r e s}$ es el periodo resonante y $Q_{\text {res }}$ la respuesta máxima medidos en la función de transferencia del sistema.

\section{Ductilidad efectiva del sistema}

En diseño sismorresistente es más razonable incluir los efectos inelásticos de interacción que los elásticos. Para ello, nosotros postulamos que la respuesta no lineal de la estructura con base flexible es aproximadamente igual a la de un oscilador equivalente con base rígida, con la misma masa que la estructura y cuyos periodo natural y fracción de amortiguamiento son iguales al periodo y amortiguamiento efectivos del sistema para la condición elástica. Para completar la caracterización de este oscilador de reemplazo, enseguida se define el concepto de ductilidad efectiva.

Las relaciones fuerza-deformación para los elementos resistentes de la estructura dada y del oscilador equivalente se muestran en la fig. 3. En estos diagramas de resistencia, $U_{y}$ y $\widetilde{U}_{y}$ denotan la deformación de fluencia de la estructura real y del oscilador de reemplazo, respectivamente, mientras que $U_{m}$ y $\widetilde{U}_{m}$ indican las correspondientes deformaciones máximas absolutas. Consecuentemente, los factores de ductilidad se definen en cada caso como $\mu_{e}=U_{m} / U_{y}$ y $\tilde{\mu}_{e}=\widetilde{U}_{m} / \widetilde{U}_{y}$.

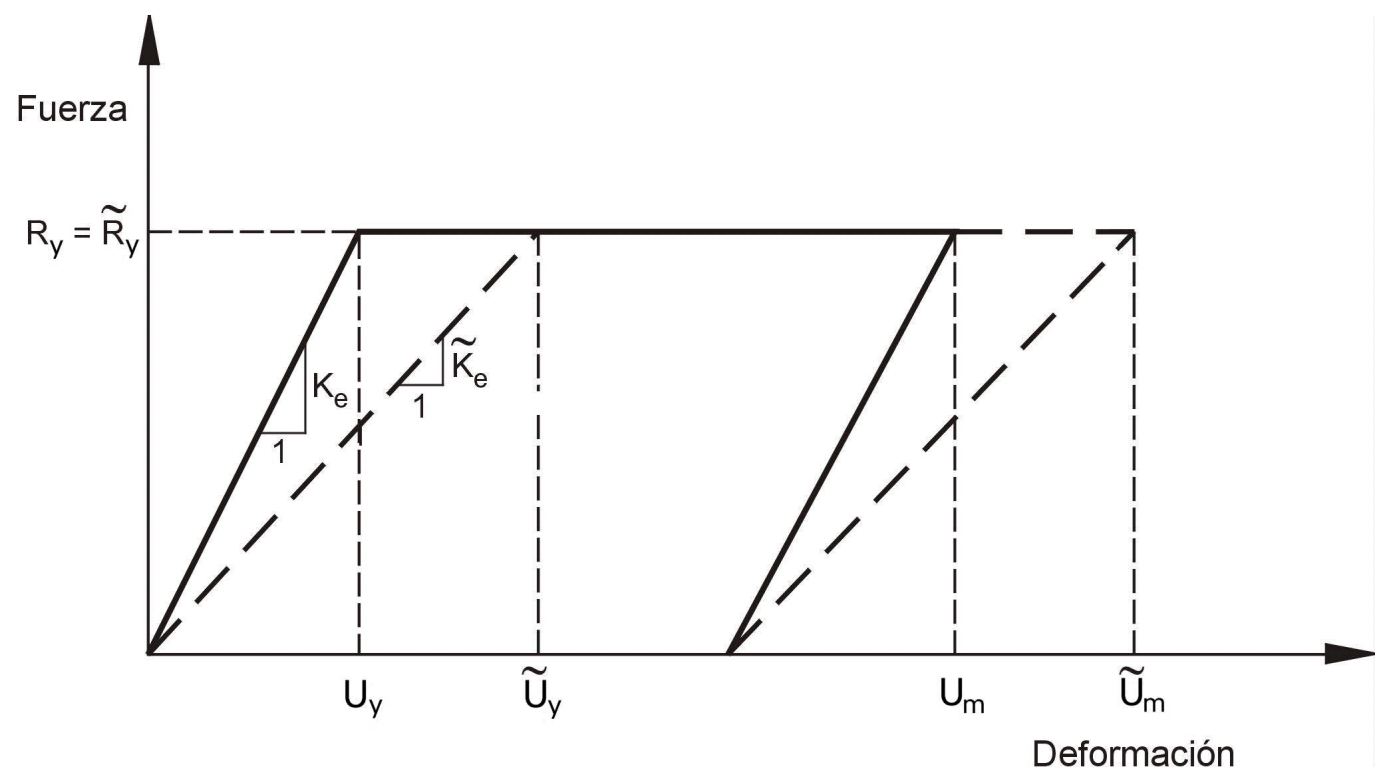

Figura 3. Relaciones fuerza-deformación para la estructura real (línea continua) y el oscilador de reemplazo (discontinua), considerando comportamiento elastoplástico.

En vista de que la resistencia de fluencia del oscilador equivalente es la misma que la de la estructura dada, se tiene que la deformación de fluencia de esta última se relaciona con la primera mediante 


$$
U_{y}=\frac{\widetilde{K}_{e}}{K_{e}} \widetilde{U}_{y}
$$

donde $\widetilde{K}_{e}=4 \pi^{2} M_{e} / \widetilde{T}_{e}^{2}$. Considerando la ec. 1, esta expresión puede escribirse alternativamente de la forma

$$
U_{y}=\frac{T_{e}^{2}}{\widetilde{T}_{e}^{2}} \widetilde{U}_{y}
$$

La capacidad de deformación plástica del oscilador equivalente y de la estructura dada deben ser idénticas. Por tanto, igualando la disipación de energía plástica de ambos elementos resistentes, se tiene

$$
\left(\widetilde{U}_{m}-\widetilde{U}_{y}\right) \widetilde{R}_{y}=\left(U_{m}-U_{y}\right) R_{y}
$$

Sustituyendo la ec. 19 en la ec. 20, considerando que $\widetilde{R}_{y}=R_{y}$ y recurriendo a las definiciones de $\mu_{e}$ y $\tilde{\mu}_{e}$, se tiene la ductilidad efectiva del sistema

$\widetilde{\mu}_{e}=\frac{T_{e}^{2}}{\widetilde{T}_{e}^{2}}\left(\mu_{e}-1\right)+1$

Esta expresión, derivada por otro medio por Rosenblueth y Reséndiz (1988), controla el comportamiento plástico del oscilador de reemplazo. De acuerdo con la ec. 21 , se tiene que $1 \leq \widetilde{\mu}_{e} \leq \mu_{e}$, lo que implica que la ductilidad efectiva del sistema es menor que la ductilidad permisible de la estructura. La ductilidad efectiva $\tilde{\mu}_{e}$ será igual a la ductilidad estructural $\mu_{e}$ para suelo infinitamente rígido e igual a la unidad para suelo infinitamente flexible. Partiendo de que $\tilde{\mu}_{e}<\mu_{e}$, podría pensarse en la aparición de menor daño estructural debido a la interacción sueloestructura. Esto no es así; ambas ductilidades implican exactamente el mismo nivel de daño, ya que la deformación plástica que el oscilador equivalente acumula durante los ciclos de histéresis se supone que es la misma que la de la estructura real.

Más adelante se mostrará que, para el caso de excitación sísmica, la reducción de la ductilidad debido a la interacción suelo-estructura también es predicha eficientemente por la ec. 21, excepto para periodos naturales extremadamente cortos. Se mostrará además que la máxima respuesta inelástica de una estructura apoyada flexiblemente puede estimarse a partir de espectros de respuesta estándar para el movimiento de campo libre especificado, empleando los valores de los parámetros $\widetilde{T}_{e}, \widetilde{\zeta}_{e}$ y $\widetilde{\mu}_{e}$ que caracterizan completamente al oscilador de reemplazo inelástico. 


\section{FUNCIONES DE IMPEDANCIA Y MOVIMIENTOS DE ENTRADA}

El cálculo de funciones de impedancia y movimientos de entrada para la cimentación es fundamental en el análisis de interacción suelo-estructura. Las funciones de impedancia se obtienen haciendo uso de una técnica eficiente de elemento finito basada en el método del estrato delgado (Tassoulas y Kausel, 1983). La importancia práctica de utilizar una técnica numérica rigurosa es que la profundidad de enterramiento y el espesor del estrato afectan significativamente a los resortes y amortiguadores con los que se reemplaza al suelo. Probablemente, el efecto más importante es que el amortiguamiento por radiación no se activa para frecuencias menores que las frecuencias críticas del estrato, definidas éstas por las frecuencias fundamentales en vibración transversal y vertical, según se trate de oscilaciones de la cimentación en traslación horizontal o cabeceo, respectivamente (Meek y Wolf, 1991). Habiendo conocido las funciones de impedancia, el movimiento de entrada se determina aplicando el método promediador de Iguchi (1982). Con esta eficiente técnica aplicable a cimentaciones enterradas, los componentes de dicho movimiento se obtienen tomando un promedio pesado de los desplazamientos de campo libre en la interfaz suelo-cimentación y sumando el desplazamiento y la rotación causados por la fuerza y el momento resultantes asociados a las tracciones de campo libre en esta superficie.

El movimiento de entrada para la cimentación bajo excitación armónica se expresa en términos de las funciones de transferencia $T_{h}(\omega)=U_{o}(\omega) / U_{g}(\omega)$ y $T_{r}(\omega)=\Phi_{o}(\omega) / U_{g}(\omega)$, que relacionan las amplitudes complejas de los movimientos de entrada horizontal y rotacional, respectivamente, entre la amplitud compleja del movimiento de control de campo libre en la superficie del terreno. Esta última amplitud se definen como (Wolf, 1985)

$$
U_{g}=\frac{2 A_{o}}{\cos \left(k_{s} H_{s}\right)+i p \operatorname{sen}\left(k_{s} H_{s}\right)}
$$

donde $p=\rho_{s} \beta_{s} / \rho_{o} \beta_{o}$ es el contraste de impedancias entre el estrato y la roca basal, mientras que $k_{s}=\omega / \beta_{s}$ es el número de onda del estrato. Sin perder generalidad, se puede aceptar que $2 A_{o}$ es la amplitud compleja del movimiento de campo libre en el afloramiento, es decir, sobre la superficie del terreno en ausencia del estrato. Por su parte, el parámetro $p$ expresa la atenuación geométrica en el estrato debido a la radiación de ondas dentro del basamento. El amortiguamiento material en el suelo se introduce a través del uso de velocidades de ondas de corte complejas, esto es: $\beta_{s, o}^{*}=\beta_{s, o}\left(1+i \zeta_{s, o}\right)$, donde los subíndices $s$ y $o$ denotan el estrato y la roca basal, respectivamente.

Conocida la respuesta armónica de la cimentación, las historias de aceleración del movimiento de entrada para un temblor en particular se pueden obtener mediante una aplicación formal de las transformadas discretas de Fourier, usando la técnica de la transformada rápida de Fourier y tomando las debidas precauciones para minimizar el error introducido en el análisis de excitaciones no periódicas. Los pasos fundamentales en el análisis de Fourier son: 1) calcular la transformada directa de Fourier, $\ddot{U}_{g}^{*}(\omega)$, del movimiento horizontal de campo libre en la 
superficie del terreno, $\left.\ddot{U}_{g}(t) ; 2\right)$ calcular las transformadas de Fourier de los componentes horizontal y rotacional del movimiento de entrada como $\ddot{U}_{o}^{*}(\omega)=T_{h}(\omega) \ddot{U}_{g}^{*}(\omega)$ y $\ddot{\Phi}_{o}^{*}(\omega)=T_{r}(\omega) \ddot{U}_{g}^{*}(\omega)$, respectivamente; y (3) calcular las historias de aceleración del movimiento de entrada, $\ddot{U}_{o}(t)$ y $\ddot{\Phi}_{o}(t)$, tomando la transformada inversa de Fourier de $\ddot{U}_{o}^{*}(\omega)$ y $\ddot{\Phi}_{o}^{*}(\omega)$. Con el movimiento efectivo de la cimentación así obtenido se procede a resolver la ec. 5.

\section{RESULTADOS NUMÉRICOS}

Los análisis se realizaron para una variedad de parámetros del sistema, a saber: para la estructura, $\mu_{e}=1,2$ y 4 y $H_{e} / R=2$ y 4 ; para el estrato, $H_{s} / R=1,2,3$ y 5 ; y para la cimentación, $D / R=0$ $\mathrm{y}$ 1. Para el resto de los parámetros se fijaron los valores $M_{c} / M_{e}=0.25, J_{c} / J_{e}=0.3$, $\rho_{s} / \rho_{o}=0.8, \beta_{s} / \beta_{o}=0.2, \zeta_{e}=\zeta_{s}=0.05, \zeta_{o}=0.03, v_{s}=0.45 \mathrm{y} v_{o}=0.33$. Estos valores se escogieron para representar a estructuras y condiciones del terreno como las que se tienen en la Ciudad de México.

Se presentan resultados para dos movimientos de control de campo libre, definidos por el terremoto de Michoacán del 19 de septiembre de 1985 (componente EW) registrado en los sitios $V I V$ de la zona de transición y SCT de la zona del lago. Las historias de aceleración de ambos registros se muestran en la fig. 4. El perfil de suelo de cada sitio se reemplazó por un estrato sencillo, cuyas propiedades se indican en la tabla 1. Los valores calculados para el periodo del sitio, es decir $T_{s}=4 H_{s} / \beta_{s}=0.6 s$ para $V I V$ y $2 s$ para $S C T$, son similares a los periodos dominantes del terreno observados. También se muestran en la fig. 4 los espectros de respuesta de pseudoaceleración normalizados con respecto a la aceleración de la gravedad, $\mathrm{Sa} / \mathrm{g}=\omega_{e}^{2} U_{y} / g$, así como los factores de reducción de resistencia $R_{\mu}$ que relacionan el espectro elástico entre los inelásticos, sin considerar los efectos de interacción suelo-estructura. Como se puede observar, las formas de $S a$ y $R_{\mu}$ están controladas por los efectos de las condiciones locales del sitio.

Tabla 1. Características del perfil de suelo en los sitios VIV y SCT

\begin{tabular}{|c|c|c|}
\hline Sitio & $\begin{array}{c}\text { Profundidad del estrato, } \\
H_{s}(m)\end{array}$ & $\begin{array}{c}\text { Velocidad promedio del suelo, } \\
\beta_{s}(\mathrm{~m} / \mathrm{s})\end{array}$ \\
\hline$V I V$ & 18 & 120 \\
\hline$S C T$ & 38 & 76 \\
\hline
\end{tabular}

\section{Demandas de ductilidad}

Se ha encontrado que la demanda de ductilidad para un temblor en particular puede relacionarse con la resistencia estructural, haciendo uso del concepto de ductilidad efectiva. Esto se ilustra con las demandas de ductilidad desarrolladas en el oscilador de reemplazo que se exhiben en la fig. 5 
para ambos sitios, en función del contraste de rigidez entre la estructura y el suelo y para diferentes combinaciones de los parámetros del sistema. La resistencia de fluencia que se proporciona al oscilador de reemplazo es la misma que la del sistema real. Los resultados predichos por la ec. 21 para la ductilidad efectiva del sistema, $\widetilde{\mu}_{e}$, muestran buena concordancia con las demandas de ductilidad $\widetilde{U}_{m} / \widetilde{U}_{y}$ del oscilador de reemplazo.
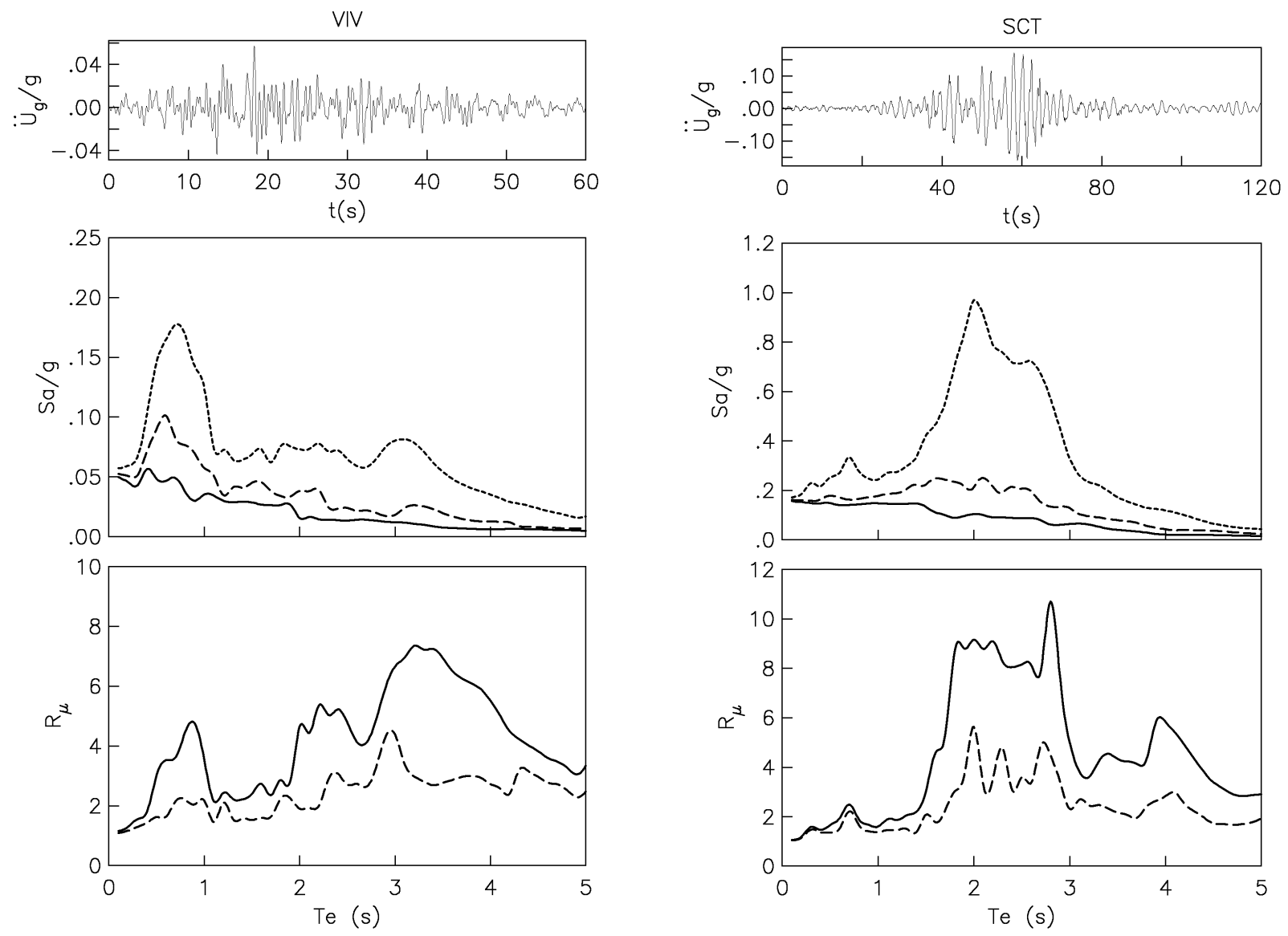

Figura 4. Arriba: acelerogramas del temblor de Michoacán de 1985 registrados en los sitios VIV y $S C T$. En medio: espectros de respuesta sin interacción para $\mu_{e}=1$ (línea punteada), 2 (discontinua) y 4 (continua). Abajo: factores de reducción de resistencia sin interacción para $\mu_{e}=2$ (línea discontinua) y 4 (continua).

Nótese que los resultados corresponden a valores fijos de $R / \beta_{s}$, como se desprende de la ec. 15 considerando que $T_{s}$ y $H_{s} / R$ están fijos. Consecuentemente, variaciones en $H_{e} / \beta_{s} T_{e}$ se deben a cambios en $T_{e}$, ya que $H_{e} / R$ también está fijo. Se puede observar que la demanda de ductilidad tiende a la ductilidad disponible para periodos naturales largos y a la unidad para periodos naturales cortos, como se predice con la ec. 21, independientemente de la configuración del sistema y de la excitación sísmica. Estos resultados también confirman que la ductilidad 
efectiva $\tilde{\mu}_{e}$ es igual a la ductilidad estructural $\mu_{e}$ para la condición límite de suelo rígido, $\beta_{s}=\infty$, e igual a la unidad para la condición límite de suelo flexible, $\beta_{s}=0$.
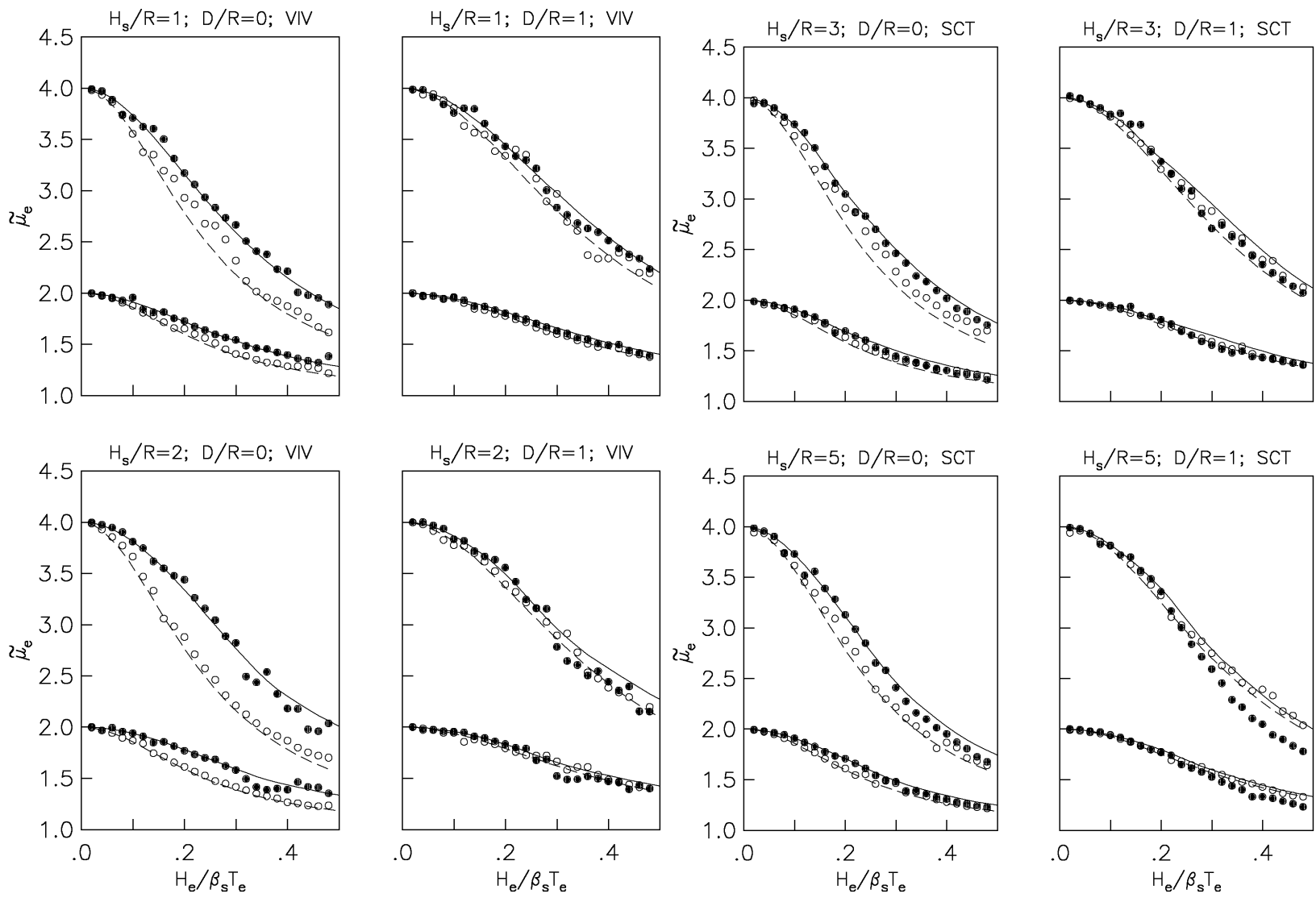

Figura 5. Comparación de la ductilidad efectiva del sistema (líneas) con la demanda de ductilidad desarrollada en el oscilador de reemplazo (círculos) para los sitios $V I V$ y $S C T$; sistemas con $\mu_{e}=2$ (curvas inferiores) y 4 (superiores) y con $H_{e} / R=2$ (línea continua y círculos llenos) y 4 (discontinua y vacíos).

La reducción de $\mu_{e}$ a $\widetilde{\mu}_{e}$ tiene lugar por el decremento de $K_{e}$ a $\widetilde{K}_{e}$, sin ningún cambio en la resistencia de fluencia ni en la capacidad de deformación plástica de la estructura con base flexible y del oscilador de reemplazo. Los resultados revelan que la reducción de la ductilidad está controlada por el contraste de rigidez entre la estructura y el suelo; mientras más flexible es la estructura o más rígido es el suelo, menor es el decremento. La influencia del resto de parámetros del sistema es relativamente menos importante. Se confirma que existe una estrecha relación entre las demandas de ductilidad desarrolladas en el oscilador de reemplazo y en la estructura con base flexible. Nótese que la ductilidad efectiva del sistema obtenida a partir de un modelo basado en la igualdad de disipación de energía plástica es independiente de la excitación sísmica. El parámetro $\widetilde{\mu}_{e}$ puede verse como la ductilidad estructural modificada por interacción suelo-estructura, tal que la resistencia de fluencia del oscilador de reemplazo sea igual a la requerida por la estructura apoyada flexiblemente para limitar la demanda de ductilidad a la 
ductilidad disponible. De esta forma, la máxima respuesta inelástica de la estructura con base flexible puede estimarse en términos del valor correspondiente de una estructura modificada con base rígida.
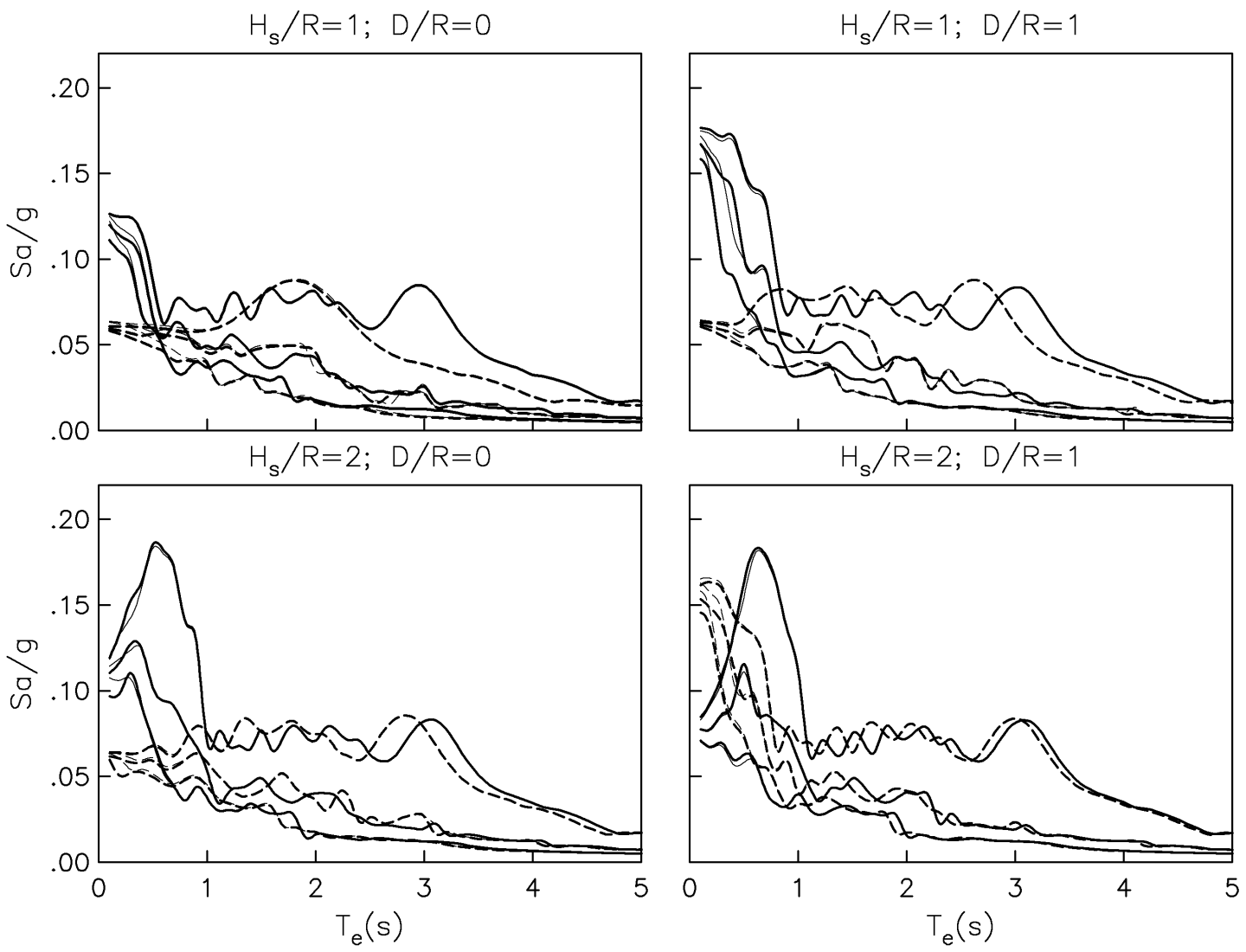

Figura 6. Espectros de respuesta con interacción para el sitio $V I V$; sistemas con $H_{e} / R=2$ (línea continua) y 4 (discontinua) y con $\mu_{e}=1,2$ y 4 (de arriba hacia abajo). Solución exacta para el sistema acoplado (línea gruesa) contra solución aproximada para el oscilador de reemplazo (delgada).

\section{Resistencias requeridas}

A continuación se presentan espectros de respuesta de ductilidad constante para diferentes combinaciones de los parámetros del sistema. Los espectros elásticos e inelásticos para los sitios $V I V$ y $S C T$ se despliegan en las figs. 6 y 7, respectivamente. En estas figuras, las abscisas representan el periodo natural de base rígida y las ordenadas representan la resistencia normalizada del sistema, $R_{y} / M_{e} g=S a / g$. Es interesante notar que la ordenada espectral para $T_{e}=0$ es independiente de los valores de ductilidad, cumpliéndose una condición teórica para estructuras rígidas. Los espectros de respuesta determinados rigurosamente para el sistema acoplado son comparados con aquéllos obtenidos aproximadamente para el oscilador de 
reemplazo. El grado de precisión de la solución aproximada es más que adecuado para aplicaciones de ingeniería. Este acuerdo confirma la eficiencia del enfoque simplificado para estimar las resistencias requeridas para estructuras apoyadas flexiblemente. El enfoque exacto se justifica sólo para estructuras inusuales de gran importancia en que los efectos de interacción tienen consecuencias definitivas en el diseño.
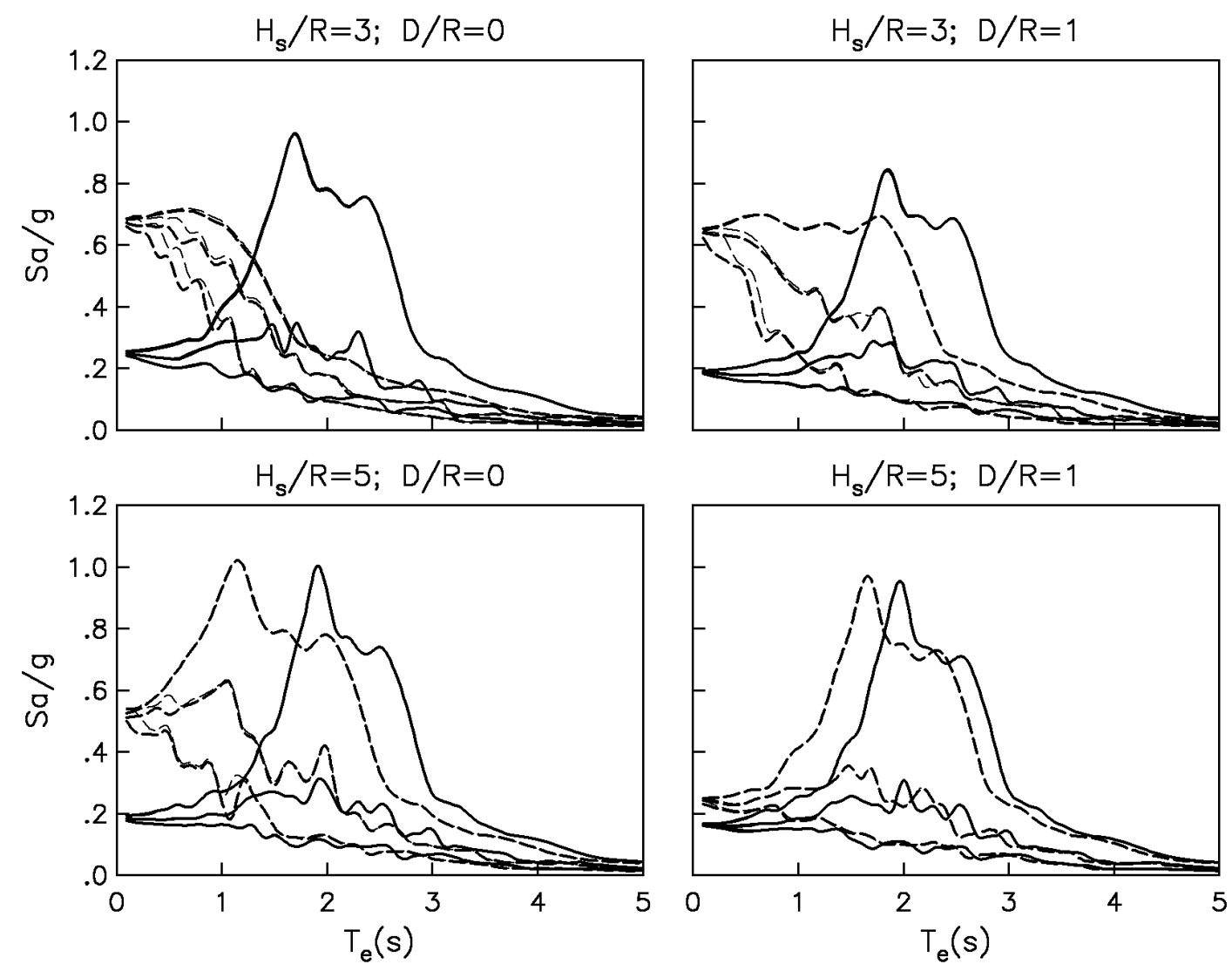

Figura 7. Espectros de respuesta con interacción para el sitio $S C T$; sistemas con $H_{e} / R=2$ (línea continua) y 4 (discontinua) y con $\mu_{e}=1,2$ y 4 (de arriba hacia abajo). Solución exacta para el sistema acoplado (líneas gruesa) contra solución aproximada para el oscilador de reemplazo (delgada).

Es necesario señalar que estos espectros de respuesta corresponden a dos relaciones de esbeltez específicas. En consecuencia, los resultados no pueden considerarse realistas en todo el intervalo de periodos naturales, ya que las estructuras con $H_{e} / R=2$ normalmente caen en la región espectral de periodos cortos, mientras que las estructuras con $H_{e} / R=4$ generalmente caen en la región de periodos largos del espectro. Fuera de estas regiones, el interés es simplemente mostrar la tendencia de los resultados. Como se sabe, el periodo de la estructura es función de su altura, de la cual depende a su vez el tamaño de la cimentación. Por tanto, los resultados sólo son válidos para las combinaciones de $H_{e}$ y $R$ que cumplan con las relaciones de esbeltez fijadas y para los valores de $T_{e}$ correspondientes. 
Las diferencias entre estos espectros de respuesta y los que se muestran en la fig. 4 reflejan los efectos de interacción suelo-estructura. Debido al alargamiento del periodo, los espectros con interacción se corren hacia la izquierda de los espectros de base rígida; mientras mayor sea el corrimiento, mayores son los efectos de interacción. A partir de estos resultados se puede concluir que, independientemente del periodo del sitio, los efectos de interacción se incrementan al aumentar $H_{e} / R$ o disminuir $D / R$ y $H_{s} / R$. Cabe señalar que para el mismo periodo del sitio, una reducción en el valor del espesor del estrato implica un incremento en el valor de la flexibilidad del suelo, como lo indica la ec. 15. Los efectos de interacción pueden traducirse en incrementos o decrementos de las resistencias requeridas, dependiendo de los valores de los parámetros del sistema, especialmente de la relación $T_{e} / T_{s}$. Generalmente, las resistencias requeridas en la región espectral $T_{e} / T_{s}<1$ llegan a ser considerablemente mayores que las calculadas si la estructura se supone apoyada rígidamente, mientras que el efecto opuesto ocurre en la región espectral $T_{e} / T_{s}>1$.
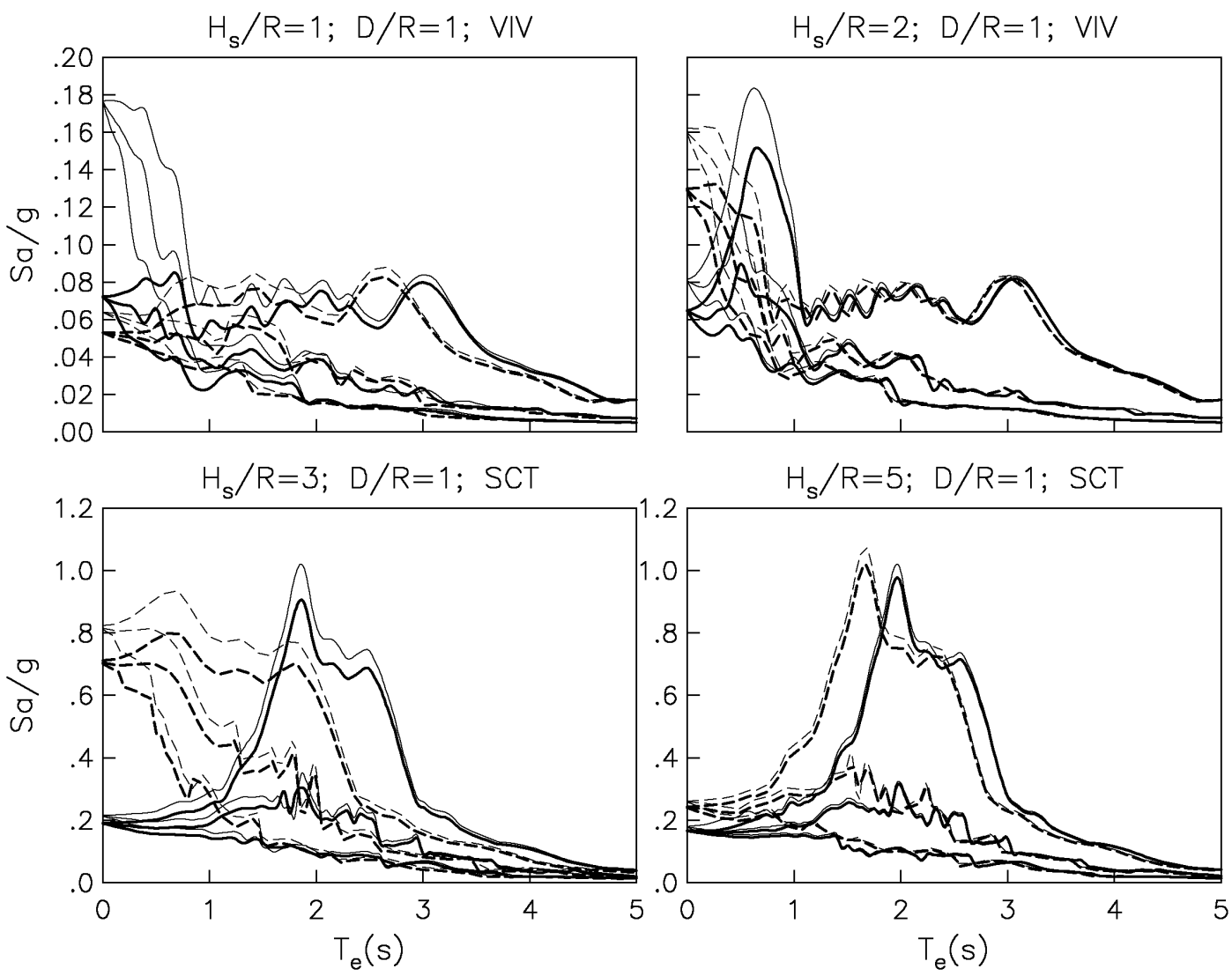

Figura 8. Comparación de espectros de respuesta considerando la interacción total (línea gruesa) con aquéllos que incluyen sólo la interacción inercial (delgada) para los sitios $V I V$ y $S C T$; sistemas con $H_{e} / R=2$ (línea continua) y 4 (discontinua) y con $\mu_{e}=1,2$ y 4 (de arriba hacia abajo). 
Algunos estudios previos de respuesta elástica empleando temblores de banda ancha (Veletsos y Meek, 1974; Avilés y Pérez-Rocha, 1998) han revelado que los efectos de interacción son significativos especialmente para estructuras rígidas de periodo corto. Puede demostrarse, sin embargo, que tales efectos también llegan a ser muy importantes para estructuras flexibles de periodo largo desplantadas en formaciones de suelo donde se originan movimientos sísmicos de banda angosta. En particular, para estructuras esbeltas con cimentación superficial que descansan sobre estratos someros, la ordenada espectral elástica para $T_{e}=T_{s}$ en el sitio $S C T$ se reduce extraordinariamente con respecto a su valor de base rígida. En este caso, los efectos de interacción suelo-estructura resultan ser tan o más significativos que los inducidos por las condiciones locales del sitio; no se puede asegurar lo mismo en el caso inelástico.

Los resultados para cimentaciones enterradas presentados en las figs. 6 y 7 para ambos sitios, ahora se comparan en la fig. 8 con los obtenidos al excluir los efectos de interacción cinemática, es decir, analizando el sistema acoplado excitado por el movimiento de campo libre aplicado en la base de la cimentación. Es claro que los efectos de interacción inercial son, en general, los más importantes. Independientemente del sitio, la interacción cinemática reduce la resistencia del sistema correspondiente a la interacción inercial solamente, particularmente para estratos someros. Como puede verse, la excitación efectiva de entrada no cambia drásticamente la forma de los espectros de respuesta calculados con el movimiento de campo libre. Estos resultados sugieren que la respuesta máxima así calculada puede considerarse como el límite superior de interacción, incluso para estructuras esbeltas que son excitadas más efectivamente por el cabeceo de la cimentación.

\section{Factores de interacción y de reducción de resistencia}

Para conocer hasta qué punto los efectos de interacción suelo-estructura difieren entre sistemas elásticos e inelásticos, se calculó el factor de interacción

$$
R_{\beta}\left(T_{e}\right)=\frac{S a\left(\mu_{e}, \beta_{s}\right)}{S a\left(\mu_{e}, \infty\right)}
$$

que relaciona los espectros de base flexible entre el de base rígida, empleando los resultados de la fig. 4 para $\beta_{s}=\infty$ y de las figs. 6 y 7 para $\beta_{s}=120$ y $76 \mathrm{~m} / \mathrm{s}$, respectivamente. Las variaciones de $R_{\beta}$ en función del periodo de la estructura se muestran en las figs. 9 y 10 para los sitios $V I V$ y $S C T$, respectivamente. Se puede ver que los efectos de interacción en sistemas inelásticos son diferentes de los que se tienen en sistemas elásticos. Desafortunadamente, estos resultados son muy sensibles a la variación del periodo de la estructura, más de lo típicamente observado en los espectros de respuesta que se emplearon para calcularlos. No es práctico tomar en cuenta este comportamiento irregular de $R_{\beta}$ en el contexto de normas de diseño sísmico. Pese a ello, pueden señalarse algunas observaciones generales: 

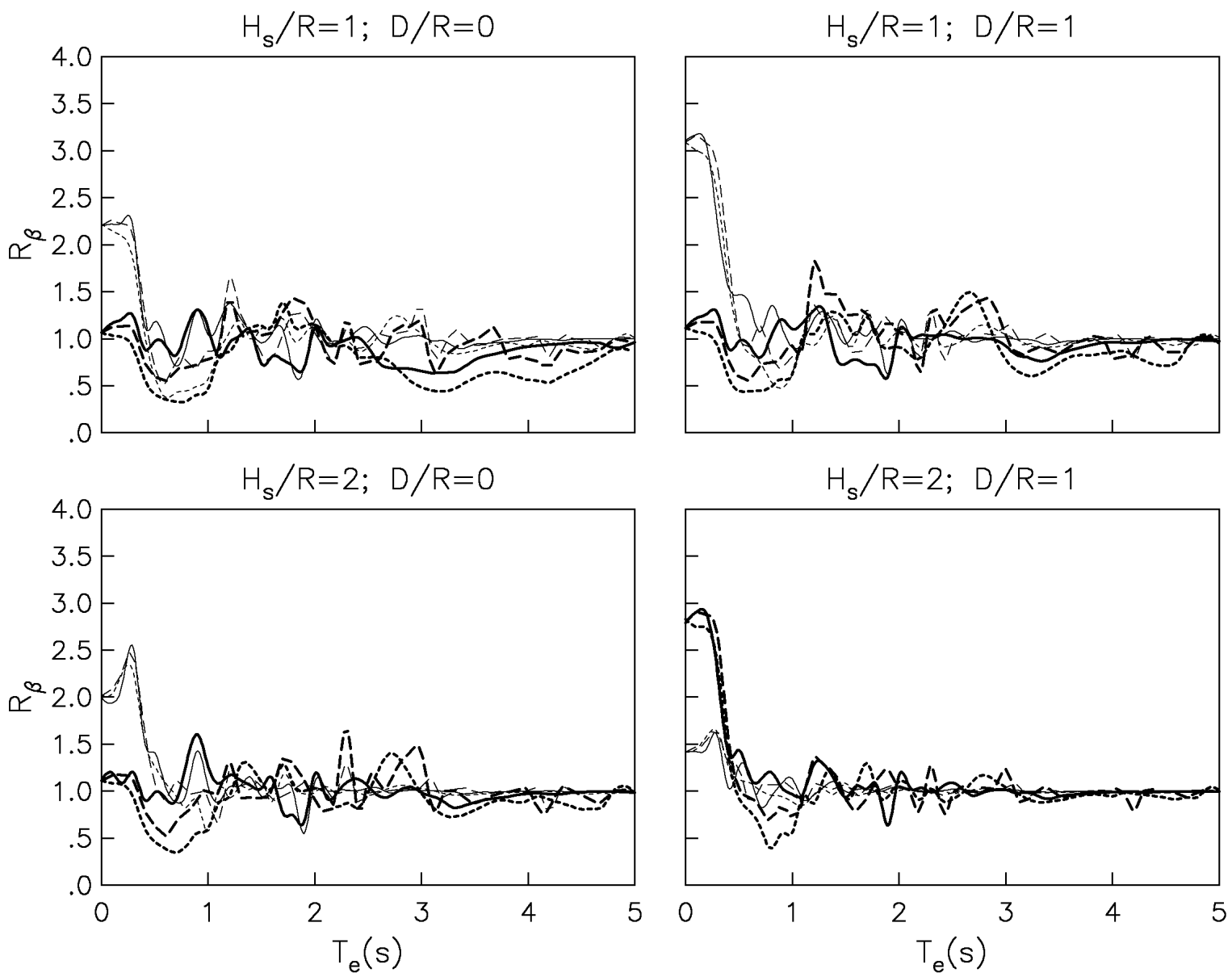

Figura 9. Variación del factor de interacción en el sitio $V I V$, para sistemas con $H_{e} / R=2$ (línea delgada) y 4 (gruesa) y con $\mu_{e}=1$ (línea punteada), 2 (discontinua) y 4 (continua).

1) La resistencia del sistema se incrementa $\left(R_{\beta}>1\right)$ en la región espectral de periodos cortos y se reduce $\left(R_{\beta}<1\right)$ en la región espectral de periodos largos. Específicamente en el sitio $S C T$, mientras más esbelta sea la estructura y menores sean los valores de $D / R$ y $H_{s} / R$, mayores serán los efectos de interacción. Las mayores reducciones e incrementos en las resistencias requeridas son del mismo orden, siendo los valores de $R_{\beta}$ alrededor de 0.25 y 4 , respectivamente. Para periodos naturales extremadamente largos, $T_{e}>5 \mathrm{~s}$, la influencia de la interacción suelo-estructura es despreciable y se tiene que $R_{\beta}=1$.

2) Para configuraciones del sistema similares, los efectos de interacción son más pronunciados en el sitio $S C T$ que en $V I V$, por ser este último sitio sensiblemente menos blando. En algunos casos sucede lo contrario, debido a que la intensidad de los efectos de interacción depende, de hecho, de la interrelación entre las características del sistema acoplado y de la excitación sísmica, y no sólo de la flexibilidad del suelo. 
3) Mientras los mayores incrementos en las resistencias requeridas ocurren en los sistemas no lineales, las mayores reducciones se presentan en los sistemas lineales. En este último caso, se debe notar que las reducciones más grandes tienen lugar cuando el periodo de la estructura es ligeramente mayor que el periodo del sitio, siendo este efecto más evidente para el sitio VIV que para $S C T$.
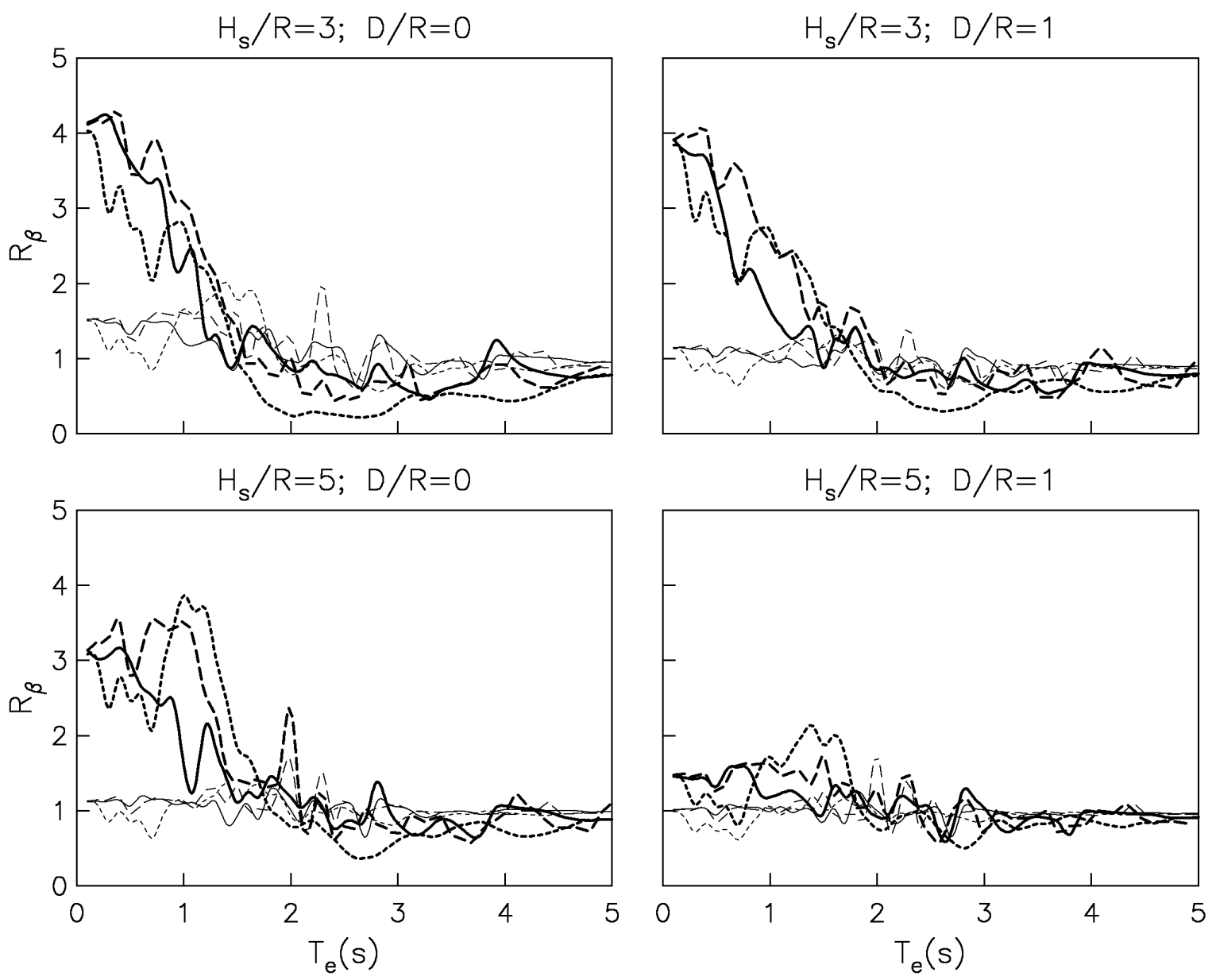

Figura 10. Variación del factor de interacción en el sitio $S C T$, para sistemas con $H_{e} / R=2$ (línea delgada) y 4 (gruesa) y con $\mu_{e}=1$ (línea punteada), 2 (discontinua) y 4 (continua).

En vista de que la interacción suelo-estructura afecta diferente a los sistemas inelásticos que a los elásticos, los factores de reducción de resistencia resultan estar influidos por la flexibilidad del suelo. Se calcularon los cocientes de las ordenadas espectrales elásticas entre las inelásticas para una ductilidad dada, como

$$
R_{\mu}\left(T_{e}\right)=\frac{S a\left(1, \beta_{s}\right)}{S a\left(\mu_{e}, \beta_{s}\right)}
$$


Los límites impuestos por la teoría para periodos naturales muy corto y largo son $R_{\mu}=1$ si $T_{e}=0$ y $R_{\mu}=\mu_{e}$ si $T_{e}=\infty$, para todas las ductilidades y sin importar la configuración del sistema y las características de la excitación sísmica. No hay indicaciones teóricas acerca de los valores de $R_{\mu}$ para periodos naturales intermedios. Es claro que la determinación de $R_{\mu}$ permite la estimación de resistencias inelásticas mediante la reducción de resistencias elásticas. Para edificios rígidamente apoyados, la regla de reducción más aceptada para diseño es la propuesta por Veletsos y Newmark (1960). Basada en la premisa de que los máximos desplazamientos elástico e inelástico son iguales, dicha regla especifica que $R_{\mu}=\mu_{e}$ si el periodo de la estructura no es excesivamente corto (ver ec. 4). Asimismo se han desarrollado reglas empíricas que incluyen los efectos de sitio, por Miranda (1993) y recientemente por Ordaz y Pérez-Rocha (1998). Son aplicables a una gran variedad de condiciones del subsuelo, pero no toman en cuenta los efectos de interacción suelo-estructura. Estos autores han mostrado que los valores de $R_{\mu}$ cuando el periodo de la estructura es parecido al periodo del sitio son notablemente mayores que los predichos por la regla de Veletsos y Newmark. Este efecto se ve claramente en los resultados de la fig. 4, especialmente para el sitio $S C T$.
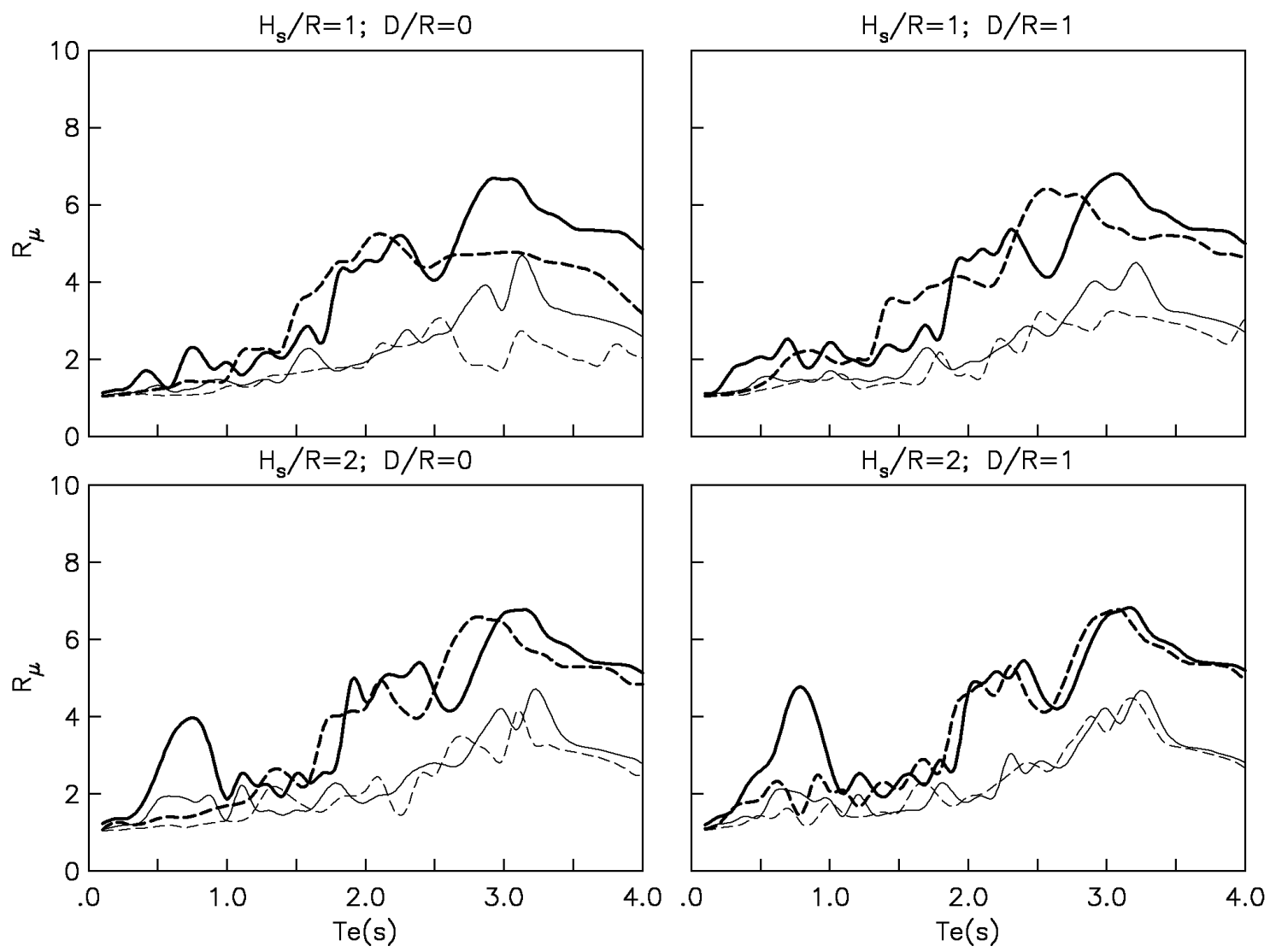

Figura 11. Variación del factor de reducción de resistencia en el sitio $V I V$ para sistemas con $H_{e} / R=2$ (línea continua) y 4 (discontinua) y con $\mu_{e}=2$ (línea delgada) y 4 (gruesa). 
De acuerdo con lo anterior, los efectos de las condiciones de sitio en las resistencias requeridas están razonablemente bien entendidos. Los efectos adicionales debidos a la interacción suelo-estructura pueden apreciarse en las figs. 11 y 12, donde se muestran las variaciones de $R_{\mu}$ en función del periodo de la estructura para los sitios VIV y SCT, respectivamente. Estos resultados se calcularon empleando los espectros de respuesta de las figs. 6 y 7 para ambos sitios. Es interesante notar que la forma de $R_{\mu}$ cambia apreciablemente tanto con el periodo natural de la estructura como con el periodo dominante del terreno. También es notable la diferencia entre los resultados para las condiciones de base flexible y apoyo rígido. En consecuencia, la reducción de espectros elásticos para obtener espectros inelásticos no procede hacerla directamente con reglas aproximadas que se han deducido suponiendo base indeformable.
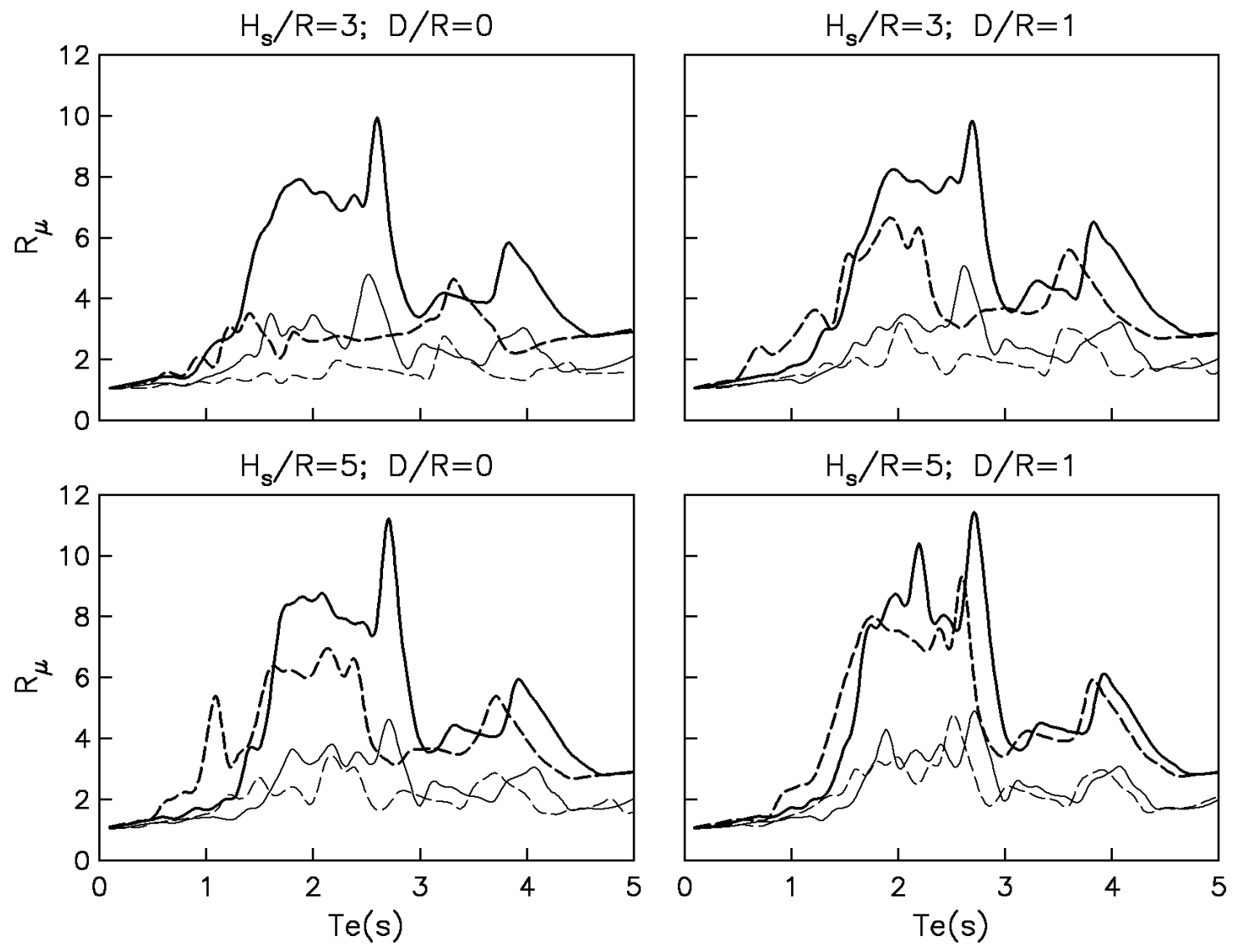

Figura 12. Variación del factor de reducción de resistencia en el sitio $S C T$, para sistemas con $H_{e} / R=2$ (línea continua) y 4 (discontinua) y con $\mu_{e}=2$ (línea delgada) y 4 (gruesa).

Si bien no es posible establecer conclusiones definitivas, cabe señalar algunas observaciones generales:

1) Los efectos de interacción resultan en valores de $R_{\mu}$ menores que los correspondientes a la condición de base rígida, siendo este hecho más evidente para el sitio SCT que para $V I V$. La 
diferencia de comportamiento entre los resultados para ambos sitios se atribuye no sólo a la flexibilidad del suelo, sino también al ancho de banda del movimiento del terreno, la cual refleja los efectos de las condiciones del subsuelo que están implícitos en la excitación sísmica seleccionada para cada sitio.

2) Un parámetro crucial es la relación de esbeltez de la estructura. Para estructuras con $H_{e} / R=2$, la forma de $R_{\mu}$ es similar a la que se obtiene para la condición de base rígida, sin importar mucho los valores de $D / R$ y $H_{s} / R$. En este caso, las resistencias requeridas pueden ser bien predichas por las reglas de reducción que incluyen exclusivamente los efectos de sitio (Miranda, 1993; Ordaz y Pérez-Rocha, 1998). Los valores de $R_{\mu}$ para estructuras con $H_{e} / R=4$ son los más afectados por interacción. En particular, para cimentaciones superficiales sobre estratos someros, los valores más grandes de $R_{\mu}$ son del orden de 2 para $\mu_{e}=2$ y de 4 para $\mu_{e}=4$. Al parecer, para este caso, la regla de Veletsos y Newmark sorprendentemente funciona mejor que las reglas de reducción dependientes de las condiciones de sitio.

\section{CONCLUSIONES}

Se ha hecho una evaluación de los efectos de interacción suelo-estructura en las resistencias requeridas para sistemas inelásticos, empleando una analogía con un oscilador elastoplástico de base rígida, definido por una ductilidad efectiva en adición al periodo y amortiguamiento efectivos del sistema para la condición elástica. La eficiencia de este oscilador de reemplazo para calcular las resistencias inelásticas requeridas se validó por comparación con resultados obtenidos rigurosamente para el sistema acoplado. Los efectos de interacción cinemática e inercial se examinaron para un amplio intervalo de los principales parámetros involucrados. Los resultados muestran que los efectos cinemáticos son de poca importancia práctica. En cambio, las diferencias entre los efectos inerciales en sistemas elásticos e inelásticos pueden ser bastante significativas. En general, los efectos de interacción se incrementan cuando aumenta $H_{e} / R$ o disminuyen $D / R$ y $H_{s} / R$. La resistencia estructural puede experimentar grandes incrementos para $T_{e} / T_{s}<1$ así como grandes reducciones para $T_{e} / T_{s}>1$, con respecto a su valor de base rígida. Los efectos del comportamiento inelástico de la estructura se evaluaron mediante factores de reducción de resistencia, cuyas formas sugieren modificar las reglas de reducción para diseño desarrolladas bajo el supuesto de apoyo indeformable.

Con la información que se ha presentado, el análisis no lineal simplificado de interacción puede realizarse eficientemente como sigue: 1) considerando la interacción cinemática e inercial, determínense el periodo y amortiguamiento efectivos del sistema para la condición elástica, $\widetilde{T}_{e} \mathrm{y}$ $\widetilde{\zeta}_{e}$, con las ecs. 16 y 17 , respectivamente; 2 ) estímese la ductilidad efectiva del sistema, $\widetilde{\mu}_{e}$, con la ec. 21 ; 3) usando espectros de respuesta estándar de campo libre, obténgase la resistencia de fluencia del sistema, $R_{y}$, correspondiente a $\widetilde{T}_{e}, \widetilde{\zeta}_{e}$ y $\widetilde{\mu}_{e}$, tal como si la estructura modificada 
estuviera rígidamente apoyada; y (4) si se desea, calcúlese la deformación máxima de la estructura como $U_{m}=\mu_{e} U_{y}$, siendo $U_{y}=R_{y} / K_{e}$.

El enfoque de diseño actualmente en uso en los reglamentos sísmicos para edificios no considera los efectos de interacción en la ductilidad. Sigue esencialmente los mismos pasos descritos arriba para determinar la resistencia requerida y el desplazamiento esperado, excepto el paso 2. Estas cantidades se obtienen usando espectros de respuesta elástica junto con los valores

de $\widetilde{T}_{e}$ y $\widetilde{\zeta}_{e}$, reducidos por ductilidad con el factor $R_{\mu}$ correspondiente a base indeformable. Hemos mostrado, sin embargo, que la diferencia entre los factores de reducción de resistencia para las condiciones de base flexible y apoyo rígido puede ser muy importante. A pesar de la simplicidad del oscilador elastoplástico investigado, sigue siendo la base de la práctica del diseño actual. De ahí que las conclusiones obtenidas en este estudio podrían ser aplicables a estructuras de edificios con comportamiento plástico.

\section{REFERENCIAS}

Applied Technology Council (1984), Tentative Provisions for the Development of Seismic Regulations for Buildings, ATC-3-06, California.

Avilés, J y L E Pérez-Rocha (1996), "Evaluation of interaction effects on the system period and the system damping due to foundation embedment and layer depth", Soil Dynamics and Earthquake Engineering, Vol. 15, pp. 11-27.

Avilés, J y L E Pérez-Rocha (1998), "Effects of foundation embedment during building-soil interaction”, Earthquake Engineering and Structural Dynamics, Vol. 27, pp. 1523-1540.

Avilés, J, M Suárez y F J Sánchez-Sesma (2002), "Effects of wave passage on the relevant dynamic properties of structures with flexible foundation", Earthquake Engineering and Structural Dynamics, Vol. 31, pp. 139-159.

Bazán, E, I Díaz, J Bielak y N Bazán (1992), "Probabilistic seismic response of inelastic building foundation systems", Memorias, $10^{\text {th }}$ World Conference on Earthquake Engineering, Madrid.

Bielak, J (1975), "Dynamic behavior of structures with embedded foundations", Earthquake Engineering and Structural Dynamics, Vol. 3, pp. 259-274.

Bielak, J (1978), "Dynamic response of non-linear building-foundation systems", Earthquake Engineering and Structural Dynamics, Vol. 6, pp. 17-30.

Federal Emergency Management Agency (1994), Recommended Provisions for the Development of Seismic Regulations for New Buildings, NEHRP-BSSC, Washington.

Iguchi, M (1982), “An approximate analysis of input motions for rigid embedded foundations", Trans. of Architectural Institute of Japan, No. 315, pp. 61-75.

Jennings, P C y J Bielak (1973), "Dynamics of building-soil interaction”, Bulletin of the Seismological Society of America, Vol. 63, pp. 9-48. 
Meek, J W y J P Wolf (1991), "Insights on cutoff frequency for foundation on soil layer", Earthquake Engineering and Structural Dynamics, Vol. 20, pp. 651-665.

Miranda, E (1993), "Site-dependent strength reduction factors", ASCE Journal of Structural Engineering, Vol. 119, pp. 3503-3519.

Ordaz, M y L E Pérez-Rocha (1998), "Estimation of strength-reduction factors for elastoplastic systems: a new approach", Earthquake Engineering and Structural Dynamics, Vol. 27, pp. 889901.

Rodríguez, M E y R Montes (1998), “Comportamiento sísmico no lineal de edificaciones sobre suelo blando", Revista de Ingeniería Sísmica, SMIS, No. 58, pp. 1-20.

Rosenblueth, E y D Reséndiz (1988), "Disposiciones reglamentarias de 1987 para tener en cuenta interacción dinámica suelo-estructura", Series del Instituto de Ingeniería No. 509, Universidad Nacional Autónoma de México.

Tassoulas, J L y E Kausel (1983), "Elements for the numerical analysis of wave motion in layered strata", International Journal for Numerical Methods in Engineering, Vol. 19, pp. 10051032.

Todorovska, M I y M D Trifunac (1992), "The system damping, the system frequency and the system response peak amplitudes during in-plane building-soil interaction", Earthquake Engineering and Structural Dynamics, Vol. 21, pp. 127-144.

Veletsos, A S y N M Newmark (1960), "Effect of inelastic behavior on the response of simple systems to earthquake motions", Memorias, $2^{\text {nd }}$ World Conference on Earthquake Engineering, Tokio.

Veletsos, A S, N M Newmark y C V Chelapati (1965), "Deformation spectra for elastic and elastoplastic systems subjected to ground shock and earthquake motions", Memorias, $3^{\text {rd }}$ World Conference on Earthquake Engineering, Wellington.

Veletsos, A S (1969), "Maximum deformations of certain nonlinear systems", Memorias, $4^{\text {th }}$ World Conference on Earthquake Engineering, Santiago.

Veletsos, A S y W P Vann (1971), "Response of ground-excited elastoplastic systems", ASCE Journal of the Structural Division, Vol. 97, pp. 1257-1281.

Veletsos, A S y J W Meek (1974), "Dynamic behaviour of building-foundation systems", Earthquake Engineering and Structural Dynamics, Vol. 3, pp. 121-138.

Veletsos, A S y B Verbic (1974), "Dynamics of elastic and yielding structure-foundation systems", Memorias, $5^{\text {th }}$ World Conference on Earthquake Engineering, Roma.

Wolf, J P (1985), Dynamic Soil-Structure Interaction, Prentice-Hall, New Jersey.

Wolf, J P y D R Somaini (1986), “Approximate dynamic model of embedded foundation in time domain”, Earthquake Engineering and Structural Dynamics, Vol. 14, pp. 683-703.

Wolf, J P (1988), Soil-Structure Interaction Analysis in Time Domain, Prentice-Hall, New Jersey. 